\title{
Function Analysis of Industrial Robot under Cubic Polynomial Interpolation in Animation Simulation Environment
}

\author{
You Li ${ }^{1}$, Juan Wang ${ }^{1}$, Yiming Ji2* \\ ${ }^{1}$ School of Arts Design, Wuchang University of Technology, Wuhan (China) \\ ${ }^{2}$ School of Journalism, Zhongnan University of Economics and Law, Wuhan (China) \\ Received 7 May 2020 | Accepted 28 October 2020 | Published 25 November 2020
}

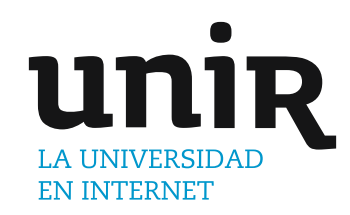

KEYWORDS

Polishing Robot Operating Arm, Cubic Polynomial Interpolation, Trajectory Planning, Kinematic Model, Multi-body Dynamics Simulation Software.

In order to study the effect of cubic polynomial interpolation in the trajectory planning of polishing robot manipulator, firstly, the articular robot operating arm is taken as the research object, and the overall system of polishing robot operating arm with 7 degrees of freedom is constructed. Then through the transformation of space motion and pose coordinate system, Denavit-Hartenberg (D-H) Matrix is introduced to describe the coordinate direction and parameters of the adjacent connecting rod of the polishing robot, and the kinematic model of the robot is built, and the coordinate direction and parameters of its adjacent link are described. A multi-body Dynamic simulation software, Automatic Dynamic Analysis of Mechanical Systems (ADAMS), is used to analyze the kinematic simulation of the robot operating arm system. Finally, the trajectory of the robot manipulator is planned based on the cubic polynomial difference method, and the simulation is verified by Matrix Laboratory (MATLAB). Through calculation, it is found that the kinematic model of polishing robot operating arm constructed in this study is in line with the reality; ADAMS software is used to generate curves of the rotation angles of different joint axes and the displacement of end parts of the polishing robot operating arm changing with time. After obtaining relevant parameters, they are put into the kinematic equation constructed in this study, and the calculated position coordinates are consistent with the detection results; moreover, the polishing robot constructed in this study can realize the functions of deburring, polishing, trimming, and turning table. MATLAB software is used to generate the simulation of the movement trajectory of the polishing robot operating arm, which can show the change curve of angle and angular velocity. The difference between the angle at which the polishing robot reaches the polishing position, the change curve of angular velocity, and the time spent before and after the path optimization is compared. It is found that after path optimization based on cubic polynomial, the change curve of the polishing robot's angle and angular velocity is smoother, and the time is shortened by $17.21 \mathrm{~s}$. It indicates that the cubic polynomial interpolation method can realize the trajectory planning of the polishing robot operating arm, moreover, the optimized polishing robot has a continuous and smooth trajectory, which can improve the working efficiency of the robot.

DOI: $10.9781 /$ ijimai.2020.11.012

\section{INTRODUCTION}

T present, China's manufacturing industry is developing rapidly,
and customers have higher and higher requirements on the
surface quality of castings. Labor costs and environmental awareness
are also increasing, which greatly limits the development of China's
foundry industry. In the process of different casting production,
sand treatment, molding, core making, and other processes have
gradually achieved the goal of automatic production and intelligent
management, but the post-processing technology of casting is still
in the stage of manual operation [1], [2]. Facing the high demand of
enterprises on the surface quality of castings, the research on casting

* Corresponding author.

E-mail address: jym20200123@163.com polishing technology and related equipment has become a big trend. The appearance of flash and burr on the surface of the casting can not only affect the appearance of the casting, but also affect the performance of the casting and even the service life of the casting [3], [4]. Therefore, polishing the surface of the casting is a very important step in the manufacturing industry.

With the continuous progress of industrial technology, enterprises have been gradually moving towards the direction of intelligence and automation. The castings polished by the robot have the advantages of better quality, higher efficiency, strong flexibility, and strong adaptability [5]. Therefore, robot polishing automatic production line and casting polishing have gradually become the best choice for enterprises in the manufacturing field. Moreover, the polishing of sports robot can reduce the risk of injury to human body, ensure the uniformity of polishing quality, improve the efficiency of operation, 
and reduce the cost [6], [7]. In recent years, experts at home and abroad have explored robot polishing equipment and its automatic polishing technology, but relatively few studies have been conducted on the polishing of large castings [8]. Polishing large castings requires a high load on the robot, while for castings with complex surface shapes, manual polishing is still required [9]. The research prospect of polishing robot is broad, and the intelligent processing system of robot is of great significance to improve the working efficiency of polishing. Therefore, it is very important to shorten the running time of the robot to improve the efficiency. Some studies have shown that polynomial interpolation can carry out the optimal time trajectory planning [10], [11]. Among them, cubic polynomial interpolation is widely used. Zubko et al. calculated the accelerated light scattering based on cubic polynomial functions and found that the interpolation method could accurately calculate the light scattering characteristics [12]. Zhang et al. showed that cubic polynomial interpolation could present a better linear relation than quadratic polynomials [13]. Moreover, a large number of studies have proved that the application of cubic polynomial interpolation in motion trajectory planning under different conditions and constraints can effectively improve the running speed [14]. Dincer et al.combined cubic polynomial interpolation with Bézier curves for the trajectory planning of 2-dof parallel structure, and found that using cubic polynomial interpolation could obtain smoother trajectory curves [15].

However, at present, the research on the path planning of seven-degree-of-freedom polishing robot by cubic polynomial interpolation is still relatively few. Therefore, firstly, the mechanism types of common industrial robot operating arms are discussed, and the polishing robot operating arm system is designed with articular robot operating arms as the research object. Through the transformation of the spatial coordinate system, the kinematic model of the polishing robot manipulator is constructed. Then, virtual simulation software is used to verify the kinematic model of the polishing robot operating arm. Finally, based on cubic polynomial interpolation, the trajectory of the polishing robot manipulator is planned. The results of this study are intended to lay a foundation for optimizing the movement trajectory of the robot's operating arm and improving its polishing efficiency.

\section{Methodology}

\section{A. Mechanism Type of industrial Robot Manipulator}

Common industrial robot operating arm structures include rectangular coordinate type, cylindrical coordinate type, polar coordinate type, and joint coordinate type, etc. [16]. Among them, the rectangular robot operating nose has the simplest structure, with only three axes in the cartesian coordinate system: the $\mathrm{X}$ axis, the $\mathrm{Y}$ axis (the horizontal motion axis), and the $\mathrm{Z}$ axis (the vertical motion axis). Articular robots operate arms that are similar to human arms, with structures that mimic the human upper and lower arms, shoulder, elbow, and wrist joints. These manipulators have 5 to 6 degrees of freedom and can touch all the points in the workspace, and their movement trajectory while working can form most of the spherical motion. The operational performance of common structural industrial robot operating arms is compared, and the results are shown in Table I. Among industrial robot operating arms with different structure types, articular robot operating arms are more compact in structure and manoeuvrable in working space. Therefore, it requires less joint torque, and its own energy consumption is low, and the joint sealing and dustproof effect is excellent. Therefore, joint coordinate robot arm is finally selected as the object of this study.
TABLE I. Performance Comparison of Operating Arms of Industrial Robots With Different Structures

\begin{tabular}{|c|c|c|c|c|}
\hline Structures & $\begin{array}{c}\text { Operational } \\
\text { complexity }\end{array}$ & $\begin{array}{c}\text { Workspace } \\
\text { size }\end{array}$ & Flexibility & Their size \\
\hline $\begin{array}{c}\text { Rectangular } \\
\text { coordinate }\end{array}$ & Easy & Small & Bad & Big \\
\hline $\begin{array}{c}\text { Cylindrical } \\
\text { coordinate }\end{array}$ & $\begin{array}{c}\text { Relatively } \\
\text { easy }\end{array}$ & $\begin{array}{c}\text { Relatively } \\
\text { big }\end{array}$ & Normal & $\begin{array}{c}\text { Relatively } \\
\text { big }\end{array}$ \\
\hline $\begin{array}{c}\text { Polar } \\
\text { coordinates }\end{array}$ & complex & $\begin{array}{c}\text { Relatively } \\
\text { small }\end{array}$ & Good & $\begin{array}{c}\text { Relatively } \\
\text { small }\end{array}$ \\
\hline $\begin{array}{c}\text { Joint } \\
\text { coordinate }\end{array}$ & $\begin{array}{c}\text { Relatively } \\
\text { complex }\end{array}$ & Big & Very good & Very small \\
\hline
\end{tabular}

\section{B. The Overall Design of Polishing Robot Operating Arm System}

There are two main methods for robot polishing and casting, which are respectively used for medium and large castings, medium and small castings. The former is the polishing tool held by the robot arm for polishing processing, while the latter is the casting held by the robot arm close to the polishing tool for polishing processing [17]. The automobile cylinder castings are taken as the polishing object, and the corresponding polishing robot structure is designed to improve the polishing efficiency of automobile manufacturing industry and reduce the cost in the production process [18]. Therefore, the robot structure with strong flexibility and simple structure should be selected. In the end, the way of polishing the casting with the polishing tool held by the robot arm to polish the automobile cylinder block is chosen. The height of each stand in the car cylinder block convex table is different, and the position distribution is not uniform. Therefore, the polishing robot arm designed in this study must have such characteristics as high motion accuracy, low return clearance, low rotational inertia, and nonvariability. Finally, the specific performance indicators are determined as follows: The grinding speed is $6000 \mathrm{rpm} / \mathrm{min}$. The grinding force is $110 \mathrm{~N}$. The quality roughness $\mathrm{Ra}$ of the cylinder surface after polishing should be equal to and less than 3.0. The arms of the polishing robot extend between 600 and $1000 \mathrm{~mm}$.

The operating arm of the polishing robot is mainly composed of the base, shoulder joint, big arm, elbow joint, forearm, and wrist joint. Moreover, the articular structure connected in series between the operating arms can best imitate the motion mode of human arms. The manipulator has 7 rotation axes, and the corresponding manipulator has 7 degrees of freedom. In the process of different coaxial rotation motions, it will receive loads in both radial and axial directions. Therefore, the connection mode of bearing between connecting rod and shaft adopts double-row angular contact ball. The specific structure of each joint of the manipulator is as follows: the shoulder joint is located at the top of the whole connecting rod drive chain and has 3 degrees of freedom (namely 3 rotating axes). The angles of different rotating axes are respectively $180^{\circ}\left(-90^{\circ} \sim+90^{\circ}\right), 180^{\circ}\left(-90^{\circ} \sim+90^{\circ}\right)$ and $210^{\circ}\left(-60^{\circ} \sim+150^{\circ}\right)$; the elbow joint is located between the upper arm and the lower arm of the robot arm, and has 2 degrees of freedom (i.e. 2 joint axes). The angles of different joint axes are $240^{\circ}\left(-180^{\circ} \sim+60^{\circ}\right)$ and $180^{\circ}\left(-90^{\circ} \sim+90^{\circ}\right)$ respectively; The wrist joint has 2 degrees of freedom (i.e. 2 joint axes), and the angles of different joint axes are all $180^{\circ}(-$ $\left.90^{\circ} \sim+90^{\circ}\right)$. The structure of the polishing robot arm designed in this study is briefly shown in Fig. 1.

In order to better meet the operating requirements of the polishing robot operating arm [19], AC servo motor is selected for this study. In addition, other joint modules include harmonic gear reducer, encoder, brake, transmission gear, and support frame. According to the actual volume of the cylinder block to be polished, the outer cylinder working space size of $350^{*} 350^{*} 350 \mathrm{~mm}^{2}$ is finally selected in this study, and the cylinder with the length and height of the bottom surface of $500 \mathrm{~mm}$ and $350 \mathrm{~mm}$ are selected as the target working space. 


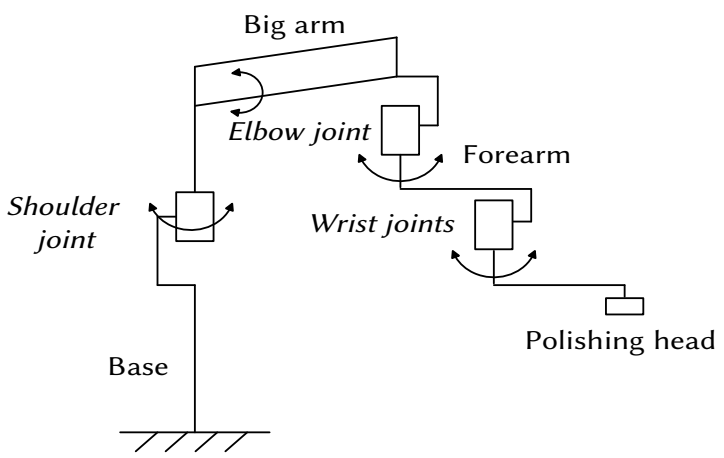

Fig. 1. Schematic diagram o polishing robot arm.

\section{The Transformation of the Spatial Coordinate System of the Operating Arm of the Polishing Robot}

Robot time - space motion is mainly divided into: firstly, forward kinematics, which refers to the relationship and parameters among the structure types and lengths of the robot's connecting rod, base and end-effector, etc, and finally, the pose problem of end-effector relative to the base coordinates is solved; secondly, inverse kinematics, it is necessary to use algebraic operation and other methods to obtain the relationship between each linkage structure, structural parameters, end-effector pose, and other information, and finally solve the variable values of each joint. In this study, the homogeneous transformation matrix method [20] is used to describe the spatial motion of the robot and the transformation relation of the linkage coordinate system, and the matrix calculation is used to solve the kinematic analysis problem of the robot manipulator.

In the spatial coordinate system, any point on the target object can be used to describe its pose. To determine the pose state of the target object on the relatively fixed coordinate system by using the $3^{*} 1$ position matrix and $3^{*} 3$ attitude matrix, the moving point coordinate system is $\{\mathrm{H}\}$, and the fixed coordinate system is $\{\mathrm{O}\}$. Then the $3^{*} 1$ position matrix can be expressed as follows.

$$
P_{0 H}=\left\{\begin{array}{l}
P_{x} \\
P_{y} \\
P_{z}
\end{array}\right\}=\left\{\begin{array}{l}
x_{0 H} \\
y_{0 H} \\
z_{0 H}
\end{array}\right\}
$$

The cosine value of the included angle between $\{\mathrm{H}\}$ and $\{\mathrm{O}\} \mathrm{x}, \mathrm{y}$, and $\mathrm{z}$ axes is used to describe the third-order attitude matrix.

$$
R_{0 H}=\left(\begin{array}{lll}
\cos \left(x_{0}, x_{H}\right) & \cos \left(x_{0}, y_{H}\right) & \cos \left(x_{0}, z_{H}\right) \\
\cos \left(y_{0}, x_{H}\right) & \cos \left(y_{0}, y_{H}\right) & \cos \left(y_{0}, z_{H}\right) \\
\cos \left(z_{0}, x_{H}\right) & \cos \left(z_{0}, y_{H}\right) & \cos \left(z_{0}, z_{H}\right)
\end{array}\right)
$$

And the initial coordinate system of any point $\mathrm{P}$ in space is $\{\mathrm{i}\}$, and the changed coordinate system is $\{\mathrm{j}\}$. In the general transformation of coordinate equation, $\mathrm{P}_{\mathrm{ij}}$ is the translation change matrix and $\mathrm{R}_{\mathrm{ij}}$ is the rotation transformation matrix. Then homogeneous coordinate transformation is introduced to describe the transformation process between coordinate systems, and finally the matrix $M_{\mathrm{ij}}$ is obtained.

$$
\begin{aligned}
M_{i j} & =\left(\begin{array}{llll}
1 & 0 & 0 & P_{x} \\
0 & 1 & 0 & P_{y} \\
0 & 0 & 1 & P_{z} \\
0 & 0 & 0 & 1
\end{array}\right)\left(\begin{array}{llll}
n_{x} & o_{z} & a_{z} & 0 \\
n_{y} & o_{y} & a_{y} & 0 \\
n_{z} & o_{z} & a_{z} & 0 \\
0 & 0 & 0 & 1
\end{array}\right) \\
M_{i j} & =\operatorname{Trans}\left(P_{x}, P_{y}, P_{z}\right) \operatorname{Rot}(k, \theta)
\end{aligned}
$$

Trans $\left(\mathrm{P}_{\mathrm{x}}, \mathrm{P}_{\mathrm{y}}, \mathrm{P}_{\mathrm{z}}\right)$ is the homogeneous coordinate transformation matrix after translation; Rot $(\mathrm{k}, \theta)$ is the homogeneous coordinate transformation matrix after rotation; $k$ is the rotation axis vector; $\theta$ is the rotation angle; $\mathrm{n}, \mathrm{o}$, and a are the cosines of the 3 directions from the coordinate system $\{\mathrm{j}\}$ to the coordinate system $\{\mathrm{i}\}$.

\section{Construction of the Kinematic Model of Polishing Robot Operating Arm}

To construct the kinematic equation of the robot manipulator, the corresponding coordinate system should be set on each revolute joint. The corresponding homogeneous coordinate system equation is obtained from the relative position of each coordinate system, and then the kinematics equation of the robot manipulator is obtained [21]. In this study, D-H method is introduced to describe the coordinate direction and parameters of the adjacent connecting rod of the robot manipulator. The schematic diagram of connecting rod parameters of robot operating arm is shown in Fig. 2. The $d_{j}, \theta_{j}, a_{j}$ and $\alpha_{j}$ in Fig. 2 are four parameters of $\mathrm{D}-\mathrm{H}$.

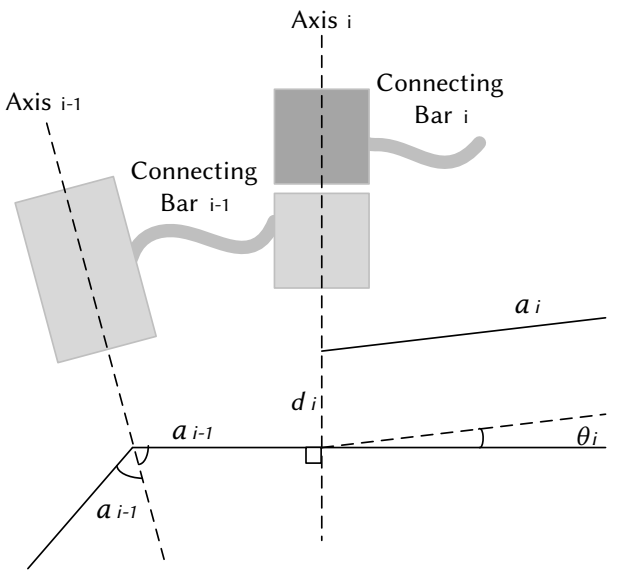

Fig. 2. Schematic diagram of parameters of adjacent connecting rod of robot operating arm.

Then in the $d-h$ coordinate system, the pose matrix between adjacent links is as follows.

$$
M_{i j}=\operatorname{Trans}\left(0,0, d_{j}\right) \operatorname{Rot}\left(z_{j-1}, \theta_{j}\right) \operatorname{Trans}\left(a_{j}, 0,0\right) \operatorname{Rot}\left(x_{j}, \alpha_{j}\right)
$$

In Eq. (5), $d_{j}$ is linkage migration; $z_{j-1}$ is the axis; $\theta_{j}$ is the joint angle; $a_{j}$ is the length of connecting rod; $\alpha_{j}$ is the torsion Angle of the connecting rod.

According to the homogeneous coordinate transformation method, the pose of the end-effector of the manipulator arm is solved.

$$
M_{0 n}=M_{0,1} M_{1,2} M_{2,3} \cdots M_{j-1, j} \cdots M_{n-1, \mathrm{n}}
$$

D-H parameters are obtained according to the linkage coordinate system, as shown in Table II.

TABle iI. Robot Manipulator Connecting Rod D-H Parameter Table

\begin{tabular}{|c|c|c|c|c|}
\hline Connecting rod number $\mathrm{j}$ & $\theta_{\mathrm{j}}\left({ }^{\circ}\right)$ & $\left.\alpha_{\mathrm{j}}{ }^{\circ}\right)$ & $\mathrm{a}_{\mathrm{j}}(\mathrm{mm})$ & $\mathrm{d}_{\mathrm{j}}(\mathrm{mm})$ \\
\hline 1 & $\theta_{1}$ & 0 & 0 & 0 \\
\hline 2 & $\theta_{2}$ & 90 & 0 & 0 \\
\hline 3 & $\theta_{3}$ & -90 & 0 & 0 \\
\hline 4 & $\theta_{4}$ & 0 & $\mathrm{a}_{3}$ & $\mathrm{~d}_{4}$ \\
\hline 5 & $\theta_{5}$ & -90 & 0 & $\mathrm{~d}_{5}$ \\
\hline 6 & $\theta_{6}$ & 90 & 0 & 0 \\
\hline 7 & $\theta_{7}$ & 90 & 0 & 0 \\
\hline
\end{tabular}

$\mathrm{a}_{3}=450 \mathrm{~mm}, \mathrm{~d}_{4}=400 \mathrm{~mm}, \mathrm{~d}_{5}=-350 \mathrm{~mm}$.

Then, the 7 degrees of freedom is used to polish the structure characteristics of robot operating arm. Using joint variable collapse method, the 7-degree-of-freedom manipulator is transformed into a 
typical 6-degree-of-freedom manipulator. The inverse kinematics of manipulator is solved by algebraic method. The kinematic equation of the robot manipulator is as follows.

$$
\begin{aligned}
& { }_{\tau}^{0} T=\left[\begin{array}{llll}
r_{11} & r_{12} & r_{13} & P_{x} \\
r_{21} & r_{22} & r_{23} & P_{y} \\
r_{31} & r_{32} & r_{33} & P_{z} \\
0 & 0 & 0 & 1
\end{array}\right]= \\
& { }_{1}^{0} T\left(\theta_{1}\right){ }_{2}^{1} T\left(\theta_{2}\right)_{3}^{2} T\left(\theta_{3}\right)_{4}^{3} T\left(\theta_{4}\right)_{6}^{4} T\left(\theta_{6}\right)_{7}^{6} T\left(\theta_{7}\right)
\end{aligned}
$$

After a series of solutions, it is concluded that when $\theta_{5}$ is 0 , the robot manipulator is in a singular configuration, that is, the joint axis 5 and 7 are in a straight line. Therefore, when calculating $\theta_{7}$, the value of $\theta_{5}$ can be referred to.

\section{E. Virtual Simulation Analysis of Polishing Robot Operating Arm}

In this study, ADAMS is used to analyze the kinematic simulation of the polishing robot operating arm system [22]. ADAMS is a popular virtual simulation software, and its simulation analysis steps are similar to those of CAE software [23]. The specific simulation process is shown in Fig. 3, which is mainly divided into 6 steps. Step 1: preparation before modeling. The main work is the choice of path, coordinate system, unit system, and the setting of gravity acceleration. Step 2: construction of the model. The main purpose of this step is to simplify the model. Under the premise of not affecting the visual effect, only the motion-related components should be retained, which can effectively reduce the difficulty of the model and improve the accuracy of model simulation. Step 3: constrains definition. Step 4: drive application. The Time function, STEP function, and AKISPL function can be used to define the driver. Step 5: verification of the model. Before the simulation, the model needs to be tested, and the model is verified successfully if there is no under constraint or over constraint. Step 6: simulation running. The Kinematic type of interactive simulation is chosen. The simulation time is set to $25 \mathrm{~s}$ and the simulation times to 350 .

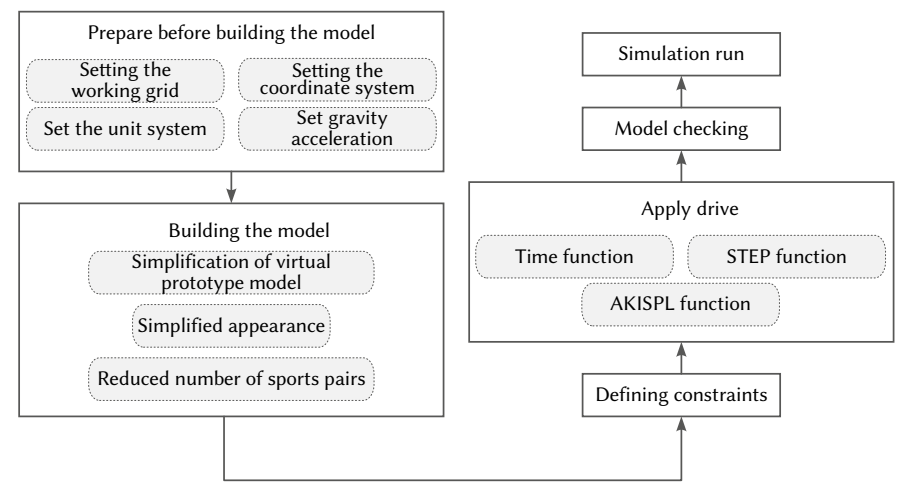

Fig. 3. Simulation process of polishing robot operating arm based on ADAMS software.

When the driver is applied, the AKISPL function needs to interpolate the data using the Akima fitting method. When the manipulator is conducting forward kinematic analysis, it is necessary to define the pose of each joint and then obtain the pose of the terminal part. Therefore, in the forward kinematics simulation of the robot operating arm, it is necessary to increase the driving force for all the 7 joint axes, so that the connecting rod outside the terminal can carry out known regular motion. Taking joint shaft 1 for example, the rotary drive diagram is chosen in the software Motions, and the rotation pair at the left and right turning joints of the shoulder is selected to create the rotation drive. Functions are input into Function(time): STEP(time, $0,0 \mathrm{~d}, 3,90 \mathrm{~d})+$ STEP(time,3,0d,6,-90d)+ STEP(time,9,0d,12,-
$90 \mathrm{~d})+$ STEP(time,12,0d,15,90d), and the type is set to Displacement. The same method is used to apply the drive to the remaining joint shafts.

\section{F. Trajectory Planning of Polishing Robot Manipulator Based on Cubic Polynomial Interpolation}

Trajectory planning is mainly to translate the command input by the operator of the robot arm into a process of describing the specific movement trajectory. Its purpose is mainly to determine the movement trajectory, movement time, speed, and other parameters of the target joint [24]. Trajectory planning of joint space mainly uses different functions to describe trajectories, and the commonly used methods are polynomial interpolation and linear interpolation based on parabolic transition [25]. In order to meet the accuracy of robot arm motion trajectory, the cubic polynomial interpolation is adopted to plan robot arm motion trajectory. Assuming that the smooth trajectory function of the joint angles at the starting and ending positions in joint space is $q(t)$, then the trajectory function $q(t)$ of different joints should meet the constraints of position and velocity. In order to ensure the continuity of joint motion speed, the starting and ending joint speeds are set to 0 . Then the trajectory function before and after can be expressed as follows.

$$
\left\{\begin{array}{l}
q(0)=q_{0} \\
q\left(t_{f}\right)=q_{f} \\
\bar{q}(0)=0 \\
\bar{q}\left(t_{f}\right)=0
\end{array}\right.
$$

In Eq. (8), $\mathrm{q}_{0}$ is the initial joint angle; $\mathrm{q}_{\mathrm{f}}$ is the joint angle of termination. $t_{\mathrm{f}}$ is the termination time.

The cubic polynomial of the constraint conditions of the boundary is determined through Eq. (8).

$$
q(t)=a_{0}+a_{1} t+a_{2} t^{2}+a_{3} t^{3}
$$

According to Eq. (9), the equations of joint velocity and acceleration are obtained.

$$
\left\{\begin{array}{l}
\bar{q}(t)=a_{1}+2 a_{2} t+3 a_{3} t^{2} \\
\overline{\bar{q}}(t)=2 a_{2}+6 a_{3} t
\end{array}\right.
$$

According to Eq. (9) and Eq. (10), different linear equations containing coefficients $\mathrm{a}_{0}, \mathrm{a}_{1}, \mathrm{a}_{2}$, and $\mathrm{a}_{3}$ are obtained.

$$
\left\{\begin{array}{l}
q_{0}=a_{0} \\
q_{f}=a_{0}+a_{1} t_{f}+a_{2} t_{f}^{2}+a_{3} t_{f}^{3} \\
0=a_{1} \\
0=a_{1}+2 a_{2} t_{f}+3 a_{3} t_{f}^{2}
\end{array}\right.
$$

By solving Eq. (11), Eq. (12) is obtained as follows.

$$
\left\{\begin{array}{l}
a_{0}=q_{0} \\
a_{1}=0 \\
a_{2}=\frac{3}{t_{f}^{2}}\left(q_{f}-q_{0}\right) \\
a_{3}=-\frac{2}{t_{f}^{3}}\left(q_{f}-q_{0}\right)
\end{array}\right.
$$

The boundary constraint condition is set as a unique cubic polynomial, and the corresponding constraint condition is substituted into Eq. (8) to obtain the corresponding linear equation. In order to ensure the continuity and stability of the motion when the critical starting and ending velocities are 0 , the cubic polynomial function can be expressed as follows.

$$
q(t)=q_{0}+\frac{3}{t_{f}^{2}}\left(q_{f}-q_{0}\right) t^{2}-\frac{2}{t_{f}^{3}}\left(q_{f}-q_{0}\right) t^{3}
$$

Then the calculation equations of joint angular velocity and angular 
acceleration are as follows.

$$
\left\{\begin{array}{l}
\bar{q}(t)=\frac{6}{t_{f}^{2}}\left(q_{f}-q_{0}\right) t-\frac{6}{t_{f}^{3}}\left(q_{f}-q_{0}\right) t^{2} \\
\overline{\bar{q}}(t)=\frac{6}{t_{f}^{2}}\left(q_{f}-q_{0}\right)-\frac{12}{t_{f}^{3}}\left(q_{f}-q_{0}\right) t
\end{array}\right.
$$

According to Eq. (14), the function curve of joint angular velocity is a parabola, while the function curve of angular acceleration is a straight line. If the angular acceleration follows the principle of constant change, it will cause the vibration of the mechanical arm, thus increasing the wear rate of the joint parts. The robot arm doesn't stay in motion at all path points, so the information of intermediate points should be considered. The path is divided into several sections, and the adjacent path points are defined as the initial point and the termination point, then the cubic polynomial function can be used for interpolation processing, and various paths are connected smoothly. Therefore, the joint velocity is not equal to 0 just for different segments. Therefore, the constraint conditions in Eq. (8) are adjusted accordingly.

$$
\left\{\begin{array}{l}
\bar{q}(0)=\bar{q}_{0} \\
\bar{q}\left(t_{f}\right)=\bar{q}_{f}
\end{array}\right.
$$

In this case, the cubic polynomial correlation equation is as follows.

$$
\left\{\begin{array}{l}
q_{0}=a_{0} \\
q_{f}=a_{0}+a_{1} t_{f}+a_{2} t_{f}^{2}+a_{3} t_{f}^{3} \\
\bar{q}_{0}=a_{1} \\
\bar{q}_{f}=a_{1}+2 a_{2} t_{f}+3 a_{3} t_{f}^{2}
\end{array}\right.
$$

The coefficients in Eq. (16) are further solved, and the results are shown in the following equation.

$$
\left\{\begin{array}{l}
a_{0}=q_{0} \\
a_{1}=\bar{q}_{0} \\
a_{2}=\frac{3}{t_{f}^{2}}\left(q_{f}-q_{0}\right)-\frac{2}{t_{f}} \bar{q}_{0}-\frac{1}{t_{f}} \bar{q}_{f} \\
a_{3}=\frac{1}{t_{f}}\left(\bar{q}_{0}+\bar{q}_{f}\right)-\frac{2}{t_{f}^{3}}\left(q_{f}-q_{0}\right)
\end{array}\right.
$$

Finally, Eq. (17) can be used to describe the trajectory of any position and velocity in the initial and final points of the path.

Then, MATLAB software is used for the simulation analysis of the motion trajectory planning of the polishing robot operating arm. The whole running time is set as $5 \mathrm{~s}$. The starting path is $\mathrm{X} 0$, the middle point $\mathrm{X} 1$ is passed through at $2.5 \mathrm{~s}$, and then the end point $\mathrm{X} 2$ is reached after $2.5 \mathrm{~s}$. At the start and end of the manipulator, the joint velocities are 0 . First, the inverse kinematics equation is used to calculate the angles of each joint at different path points, and then interpolation is performed every $20 \mathrm{~ms}$. Finally, using cubic polynomial interpolation method to plan the motion trajectory, the curve of joint axis angle with time is obtained.

\section{Results AND Discussion}

\section{A. Verification of Kinematic Solution of Operating Arm of Polishing Robot}

Since the forward kinematic solution method of the robot manipulator is relatively simple, while the reverse kinematic solution operation is complicated and there are many methods, the inverse kinematic solution method proposed has been verified by an example [26]. MATLAB software is used for verification. The corresponding solver is written first, and then the inverse solution of the end of the operating arm of the robot is obtained. Finally, the different solutions obtained are put into the forward kinematic equation to determine whether the equation is applicable. The coordinates of a target point are randomly selected as $(-150,550,-450)$, and the attitude of the end piece when the robot manipulates the arm to reach the target point is $\left(\frac{\pi}{7}, \frac{\pi}{5}, \frac{\pi}{6}\right)$. After the calculation of MATLAB software, the inverse solutions of each joint of the robot operating arm are obtained, and the results are shown in Table III. The results of 8 inverse kinematic solutions with different joint angles show the trend of arcs, and the forward kinematic equation is substituted to solve the problem.

TABLE III. Results of Inverse Kinematic Solution of Robot Manipulator Under Target Pose

\begin{tabular}{|c|c|c|c|c|c|c|c|}
\hline Grouping & $\boldsymbol{\theta}_{\mathbf{1}}\left({ }^{\circ}\right)$ & $\boldsymbol{\theta}_{2}\left({ }^{\circ}\right)$ & $\left.\boldsymbol{\theta}_{3}{ }^{\circ}{ }^{\circ}\right)$ & $\boldsymbol{\theta}_{4}\left({ }^{\circ}\right)$ & $\boldsymbol{\theta}_{5}\left(^{\circ}\right)$ & $\boldsymbol{\theta}_{\mathbf{6}}\left({ }^{\circ}\right)$ & $\boldsymbol{\theta}_{7}\left({ }^{\circ}\right)$ \\
\hline $\mathbf{1}$ & 0.752 & 1.012 & -0.230 & 1.021 & -0.563 & 0.186 & -1.689 \\
\hline $\mathbf{2}$ & 0.752 & 1.022 & 1.235 & -1.021 & -0.676 & 0.770 & -1.689 \\
\hline $\mathbf{3}$ & -1.221 & 1.233 & 1.089 & 1.021 & 0.913 & 0.182 & 0.105 \\
\hline $\mathbf{4}$ & -1.221 & -1.233 & -0.579 & -1.021 & 0.587 & 0.771 & 0.105 \\
\hline $\mathbf{5}$ & 3.879 & -1.018 & 2.920 & 1.021 & -0.563 & 0.182 & -1.689 \\
\hline $\mathbf{6}$ & 3.879 & -1.018 & 4.379 & -1.021 & -0.676 & 0.770 & -1.689 \\
\hline $\mathbf{7}$ & 1.903 & -1.231 & 4.241 & 1.021 & 0.913 & 0.186 & 0.105 \\
\hline $\mathbf{8}$ & 1.903 & 1.231 & 2.560 & -1.021 & 0.560 & 0.771 & 0.105 \\
\hline
\end{tabular}

\section{B. Polishing Robot Operating Arm Simulation Results}

Fig. 4 shows the animation simulation model of polishing robot with different performance built by ADAMS software. It can be concluded that the polishing robot needs to have the performance of deburring work built on non-smooth surface, polishing work built on medium and large scale, trimming work of complex components, and return to the working origin.
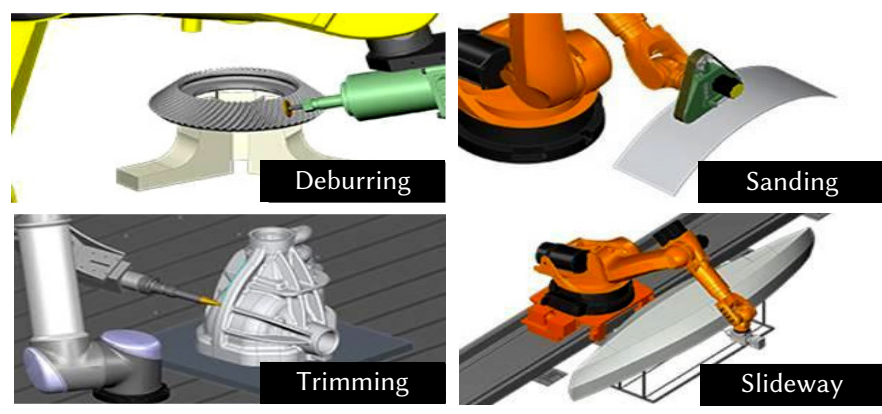

Fig. 4. The animation simulation model of polishing robot with different performance.

ADAMS software is used to process the curve graph of the output processing data of the module, so as to generate the function curve of the operating arm of the polishing robot with different coaxial changes over time to observe the specific situation of the changes of each parameter with time more intuitively. The curves of the angles of different joint axes and the displacement of end parts over time are shown in Fig. 5. It can be concluded from Fig. 5A that the change trend of the rotation Angle of axis 4 and axis 5 is basically the same, while the change trend of the rotation Angle of axis 6 and axis 7 is basically the same. As can be concluded from Fig. 5B, the initial displacement of the manipulator in the $\mathrm{X}$-axis direction (End piece1) is about $-450 \mathrm{~mm}$, and the position moves to the positive maximum at about 15s (about $600 \mathrm{~mm}$ ), and to the negative maximum at about 21s (about $-500 \mathrm{~mm}$ ). The initial displacement on the Y-axis direction (End piece2) is about $-350 \mathrm{~mm}$, and the position reaches the positive maximum at about $14 \mathrm{~s}$ (about $810 \mathrm{~mm}$ ), and the position reaches the negative maximum at about 21s (about $-500 \mathrm{~mm}$ ). The initial displacement on the z-axis direction (End piece3) is about $400 \mathrm{~mm}$, and the position reaches the maximum forward direction (about $550 \mathrm{~mm}$ ) at around $16 \mathrm{~s}$, and the position reaches the maximum negative direction (about $-810 \mathrm{~mm}$ ) at 
about 12s. As shown in Fig. 5, the movement trajectory curves of each joint axis and the end parts of the polishing robot constructed in this study present a continuous and smooth change trend, without any sudden change, which indicates that when the robot manipulators move, the joints and connecting rods present a stable and non-violent movement trend [27]. This also verifies the reliability of the mechanical structure of the polishing robot designed in this study. Finally, all can return to the starting position. The displacement of the end piece is calculated as $(550.8 \mathrm{~mm}, 400.5 \mathrm{~mm},-80.1 \mathrm{~mm})$, and the joint angles are $\theta_{1}=0^{\circ}, \theta_{2}=-90^{\circ}, \theta_{3}=-180^{\circ}, \theta_{4}=0^{\circ}, \theta_{5}=0^{\circ}, \theta_{6}=0^{\circ}, \theta_{7}=-90^{\circ}$, respectively. It is put into the kinematic equation constructed in this study, and the position coordinates obtained after calculation are consistent with the detection results, which reflects that ADAMS software is used for the simulation analysis of the polishing robot operating arm, with high accuracy and reliability [28].

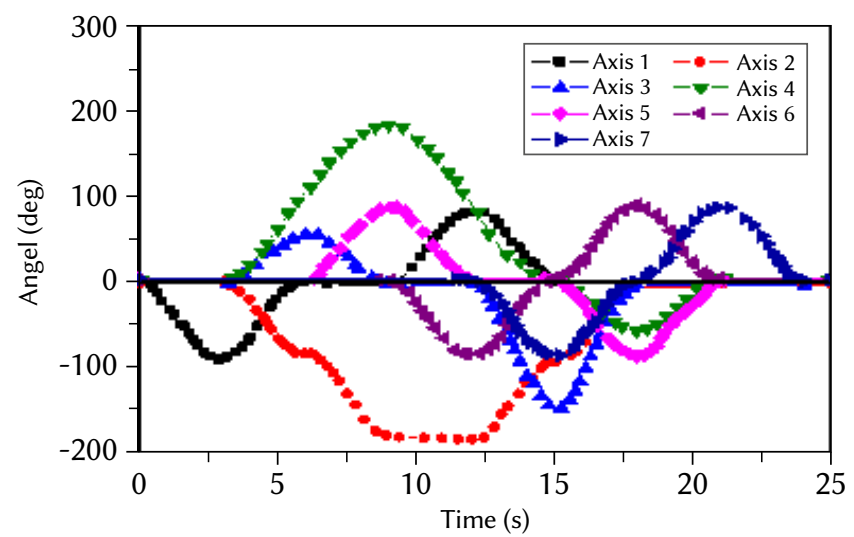

(a)

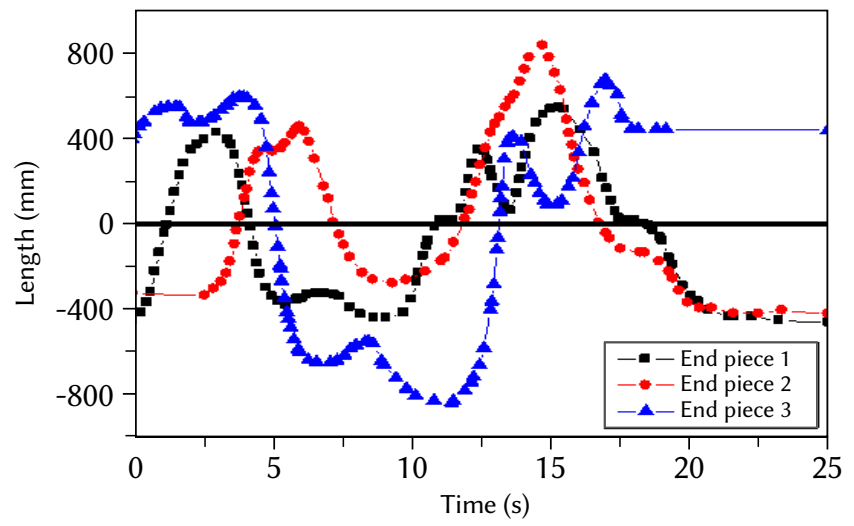

(b)

Fig. 5. The curve diagram of the rotation angle of the joint axis of the robot manipulator arm and the displacement of the terminal part with time based on ADAMS software. Note: (a) is the curve diagram of the rotation angle of joint axis changing with time; (b) is the curve graph of displacement of the terminal piece changing with time.

\section{Simulation of Trajectory Planning of the Robot Operating Arm}

MATLAB is the software often used for trajectory analysis simulation, which can be directly generated through programming data curve. The Robotic toolbox can be used to simulate track planning, and then output the relevant data curve. And for some special difference function, $\mathrm{VC}++$ and other programming can be used to automatically generate the trajectory of the simulation program [29], [30]. MATLAB software is used to generate the movement trajectory of the polishing robot operating arm. The starting point X1 coordinate is set as (17.32, $-100.00,99.09)$, the intermediate point $\mathrm{X} 2$ coordinate is $(92.17,-100.00$, $79.37)$, and the termination point $\mathrm{X} 3$ coordinate is $(54.88,-100.00$,
-100.00). The inverse kinematics equation is used to calculate the angles of each joint of the robot arm at the three path points, and the results are shown in Table IV.

TABLE IV. Joint Angles At Different Path Points

\begin{tabular}{|c|c|c|c|}
\hline Joints & Starting point $\mathrm{X}_{1}$ & Middle point $\mathrm{X}_{2}$ & End point $\mathrm{X}_{3}$ \\
\hline $1\left(^{\circ}\right)$ & -6.45 & -10.73 & -6.33 \\
\hline $2\left(^{\circ}\right)$ & -5.76 & -0.58 & 21.19 \\
\hline $3\left(^{\circ}\right)$ & -19.20 & -13.41 & 2.73 \\
\hline $4\left(^{\circ}\right)$ & 29.33 & 13.85 & -6.61 \\
\hline $5\left(^{\circ}\right)$ & -30.00 & -50.00 & -80.00 \\
\hline $6\left(^{\circ}\right)$ & -11.56 & -0.75 & 21.50 \\
\hline $7\left(^{\circ}\right)$ & 36.08 & 60.70 & 85.61 \\
\hline
\end{tabular}

After the command program is input into MARLAB software, the angle curves and angular velocity curves of each joint axis are calculated and obtained. The results are shown in Fig. 6. From the simulation curve diagram, it can be intuitively observed of the changes in the angle and angular velocity of each joint axis in the whole process from the starting point of path planning to the middle point and finally to the end point. It can be concluded from Fig. $6 \mathrm{~A}$ that the rotation angle of joint pumping 1 changes gently, while the rotation angle of joint axis 2, 3, and 7 increases gradually, and the rotation angle of joint axis 4, 5, and 6 decreases gradually. As shown in Fig. 6B, taking the time to reach the intermediate point as the node (2.5s), the angular velocity of different joint axes at this point shows obvious changes, but the angular velocity of both the starting and ending positions is 0 .

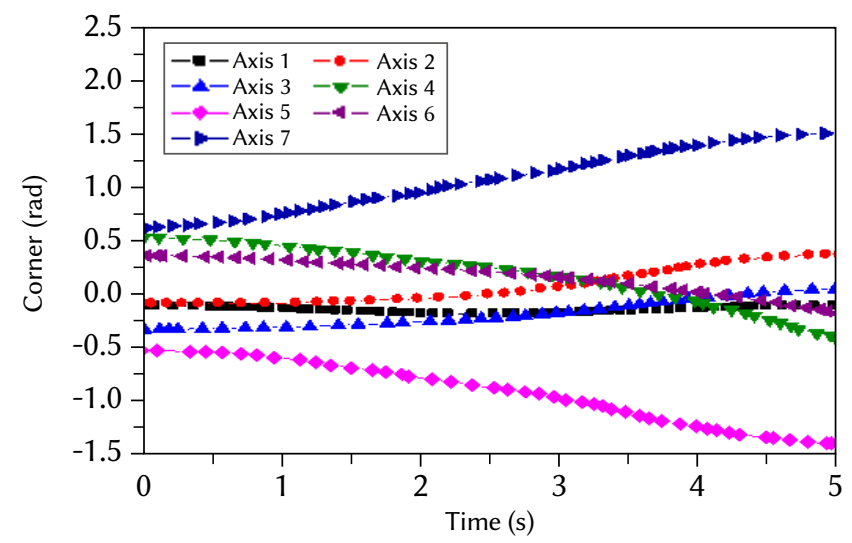

(a)

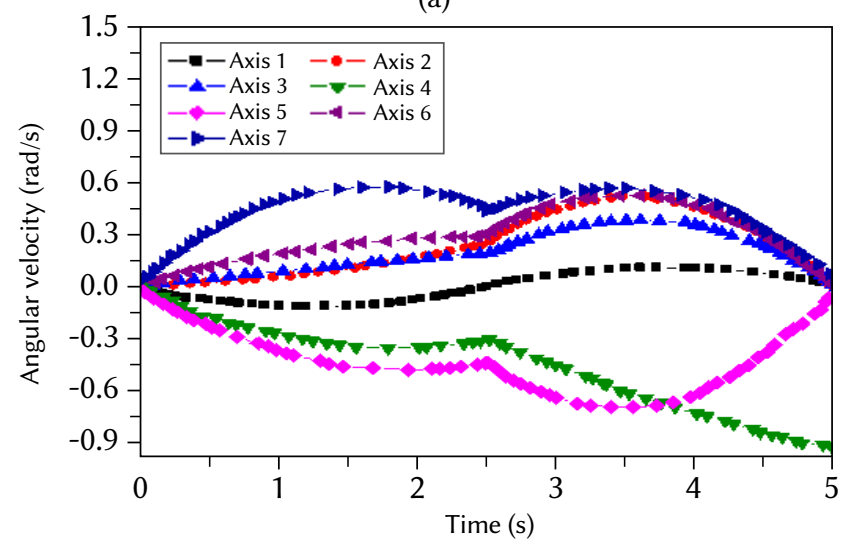

(b)

Fig. 6. The path planning simulation results of the polishing robot operating arm. Note: A is the angle curve of each joint axis of the robot operating arm; $\mathrm{B}$ is the angular velocity curve of each joint axis of the robot operating arm.

Taking joint axis 1 as an example, the change trends of angular 
displacement and angular velocity before and after optimization are compared, and the results are shown in Fig. 7. It can be concluded from Fig. 7A and Fig. 7B that under the premise of satisfying each constraint condition, the changes of angular displacement and angular velocity of the optimized joint axis 1 are continuous smooth curves. Both the angular displacement and angular velocity curves of the joint axis 1 before optimization show a certain degree of mutation, and the time to reach the maximum displacement and angular velocity of the joint axis 1 before optimization is longer than that after optimization. Based on cubic polynomial interpolation, Walambe et al. realized the planning of vehicle prototype motion trajectory, and obtained the continuous, smooth, and optimized path trajectory, which is basically consistent with the results of this study [31].

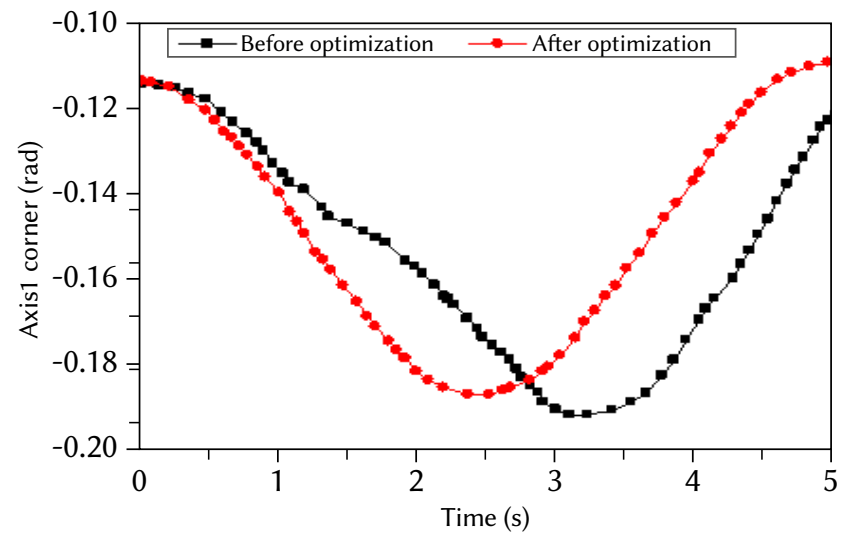

(a)

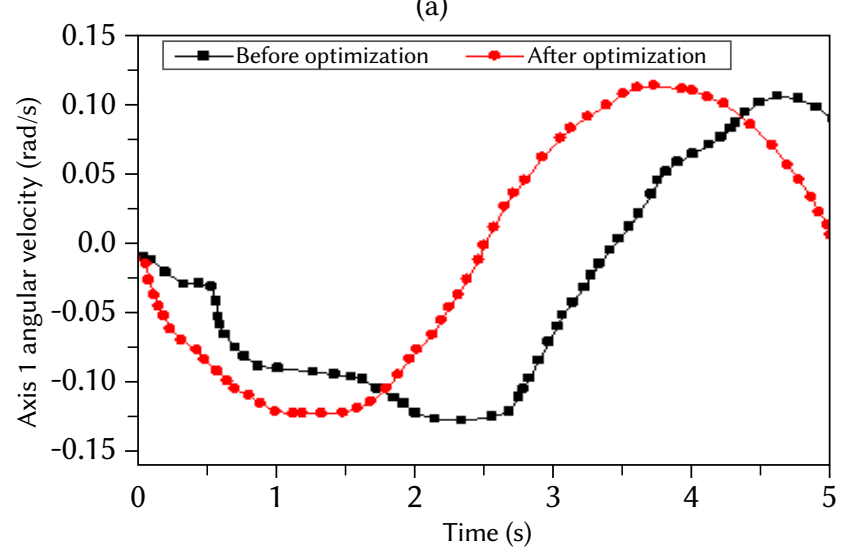

(b)

Fig. 7. Trajectory curves of joint axis 1 before and after optimization. Note: A is the angle curve of joint axis 1 before and after optimization; $\mathrm{B}$ is the angular velocity curves of joint axis 1 before and after optimization.

MATLAB software is used to simulate the movement trajectory of the polishing robot operating arm reaching the polishing target point before and after optimization, and the difference of operation time before and after optimization is compared, and the results are shown in Table V. The time for the optimized polishing robot to reach the polishing position required by the motion trajectory is $45.14 \mathrm{~s}$, while the time after the optimization is only $27.93 \mathrm{~s}$, which is shortened by about 17.21s. Moreover, the polishing position required by the trajectory after optimization is consistent with that before optimization, and there is no deviation in the position. This indicates that the trajectory planning of polishing robot based on cubic polynomial interpolation can better improve the motion speed and work efficiency of polishing robot operating arm [32]-[33].
TABle V. Comparison of the Time to Reach the Polishing Position Before ANd After Optimization

\begin{tabular}{|c|c|c|c|c|c|c|}
\hline Time $(\mathrm{s})$ & $\mathrm{t}_{1}$ & $\mathrm{t}_{2}$ & $\mathrm{t}_{3}$ & $\mathrm{t}_{4}$ & $\mathrm{t}_{5}$ & Total \\
\hline Before optimization & 7.41 & 2.38 & 15.17 & 4.99 & 15.19 & 45.14 \\
\hline After optimization & 4.22 & 3.19 & 7.29 & 3.95 & 9.28 & 27.93 \\
\hline
\end{tabular}

\section{ConClusion}

In order to explore the effect of cubic polynomial interpolation in polishing robot path optimization, in the study, firstly, a 7-degreeof-freedom articular polishing robot operating arm system is constructed. The d-h method is introduced to construct the kinematic model of the manipulator system. The kinematic model of the robot manipulator is simulated and analyzed by ADAMS software. It is found that the kinematic model of the robot operating arm is accurate. Then, the trajectory of the robot is planned based on cubic polynomial interpolation. Finally, MATLAB software is used for simulation and verification, and it is found that it could accurately show the displacement and angular velocity changes of the robot end parts. Moreover, the path planning of polishing robot is carried out using cubic polynomial interpolation method, which can effectively reduce the time to reach the polishing position compared with the unoptimized method. However, only simulation software is analyzed to carry out simulation tests, and subsequent application analysis is needed to verify the reliability of the polishing robot operating arm system proposed in this study. To sum up, the results of this study can provide theoretical basis for improving the working efficiency of polishing robot.

\section{REFERENCES}

[1] L. Song, W.H. Liu, Y.M. Li, and F.H. Xin. Humidity-resistant inorganic binder for sand core making in foundry practice, China Foundry, vol. 16, no.4, pp. 267-271, 2019, doi:10.1007/s41230-019-8169-8.

[2] Y. Watanabe, T. Sugiura, H. Sato, and H. Tsuge. Fabrication of Al-Based Composites by Centrifugal Mixed-Powder Method and Their Application for Grinding Wheels, Journal of Materials Engineering and Performance, pp. 1-12, 2019, doi:10.1007/s11665-019-03917-3.

[3] Shaofei Wu. A Traffic Motion Object Extraction Algorithm, International Journal of Bifurcation and Chaos, vol. 25, no. 14, Article Number 1540039, 2015, doi:10.1142/S0218127415400398.

[4] G. Pradeep, S. K. Kumar, G. Mathivanan, and H. Praveen. Design and Fabrication of Punch Tool Arrangement to Remove Excess Base Fillings from Aluminium Die Casting, Engineering Reports, vol. 2, no. 1, pp.3034, 2019.

[5] T. Zhang. Weighted hand-eye calibration algorithm for robot grinding, Journal of Mechanical Engineering, vol. 54, no. 17, pp. 142, 2018.

[6] K. Subrin, S. Garnier, T. Bressac, and B. Furet. Digital chain development for sanding application with a kinematically redundant robotic system, Procedia CIRP, vol. 82, pp. 515-520, 2019, doi:10.1016/j.procir.2019.04.063.

[7] H. Lyu, Y. Liu, J. Y. Guo, H. M. Zhang, and Z. X. Li. Tool-path generation for industrial robotic surface-based application, Advances in Manufacturing, vol. 7, no. 1, pp. 64-72, 2019, doi:10.1007/s40436-018-00246-x.

[8] W. X. Ng, H. K. Chan, W.K. Teo, and I.M. Chen. Capturing the tacit knowledge of the skilled operator to program tool paths and tool orientations for robot belt grinding, International Journal of Advanced Manufacturing Technology, vol. 91, no. 5-8, pp. 1599-1618, 2017, doi:10.1007/s00170-016-9813-8.

[9] D. Mahdy, R. Reda, N. Hamdi, and I.S.M. Khalil. Ultrasound-guided minimally invasive grinding for clearing blood clots: promises and challenges, IEEE Instrumentation and Measurement Magazine, vol. 21, no. 2, pp. 10-14, 2018.

[10] H. J. Lee, M. T. Soe, S. H. Chauhdary, S. Rhee, and M. S. Park. A data aggregation scheme for boundary detection and tracking of continuous objects in WSN, Intelligent Automation \& Soft Computing, vol. 23, no. 1, pp. 135-147, 2017, doi:10.1080/10798587.2016.1183922.

[11] V. Andrean, P. Chang, and K. Lian. A review and new problems discovery 
of four simple decentralized maximum power point tracking algorithmsPerturb and observe, incremental conductance, golden section search, and Newton's quadratic interpolation, Energies, vol. 11, no. 11, pp. 2966, 2018, doi:10.3390/en11112966.

[12] E. Zubko, G. Videen, J. A. Arnold, B. MacCall, A. J. Weinberger, and Y. Shkuratov. Interpolating light-scattering properties of irregularly shaped, absorbing particles, Optics letters, vol. 43, no. 17, pp. 4308-4311, 2018, doi:10.1364/OL.43.004308.

[13] B. Zhang, and Z. Feng. The Construction Method of Interpolation Polynomial by Fractal Interpolation, International Journal of Nonlinear Science, vol. 25, no. 1, pp. 48-52, 2018.

[14] Shaofei Wu, Mingqing Wang, Yuntao Zou. Research on internet information mining based on agent algorithm, Future Generation Computer Systems, vol. 86, pp. 598-602,2018, doi:10.1016/j. future.2018.04.040.

[15] Ü. Dinçer, and M. Çevik. Improved trajectory planning of an industrial parallel mechanism by a composite polynomial consisting of Bézier curves and cubic polynomials, Mechanism and Machine Theory, vol. 132, pp. 248-263, 2019, doi:10.1016/j.mechmachtheory.2018.11.009.

[16] L. Li, Y. Zhang, M. Ripperger, J. Nicho, and A. Fumagalli. Autonomous object pick-and-sort procedure for industrial robotics application, International Journal of Semantic Computing, vol. 13, no. 2, pp. 161-183, 2019, doi:10.1142/S1793351X19400075.

[17] J. R. Bourne, E.R. Pardyjak, and K.K. Leang. Coordinated bayesian-based bioinspired plume source term estimation and source seeking for mobile robots, IEEE Transactions on Robotics, vol. 35, no. 4, pp. 967-986, 2019, doi: 10.1109/TRO.2019.2912520.

[18] S. H. Kim, and J. H. Park. The Effect of Occupation-Based Bilateral Upper Extremity Training in a Medical Setting for Stroke Patients: A Single-Blinded, Pilot Randomized Controlled Trial, Journal of Stroke and Cerebrovascular Diseases, vol. 28, no. 12, pp. 104335, 2019.

[19] R. Hou, L. Wang, Q. Gao, Y. Hou, and C. Wang. Indirect adaptive fuzzy wavelet neural network with self-recurrent consequent part for AC servo system, ISA transactions, vol. 70, pp. 298-307, 2017, doi:10.1016/j. isatra.2017.04.010.

[20] H. Yan, Y. Cao, and J. Yang. Statistical tolerance analysis based on good point set and homogeneous transform matrix, Procedia Cirp, vol. 43, pp. 178-183,2016, doi:10.1016/j.procir.2016.02.042.

[21] R. R. Serrezuela, A. F. C. Chavarro, M. A. T. Cardozo, A. L. Toquica, and L. F. O. Martinez. Kinematic modelling of a robotic arm manipulator using Matlab, ARPN Journal of Engineering and Applied Sciences, vol. 12, no. 7, pp. 2037-2045, 2017.

[22] I. Pavlenko, J. Trojanowska, O. Gusak, V. Ivanov, J. Pitel, and V. Pavlenko. Estimation of the reliability of automatic axial-balancing devices for multistage centrifugal pumps, Periodica Polytechnica Mechanical Engineering, vol. 63, no. 1, pp. 52-56, 2019.

[23] X. H. Li, H. B. Yu, M. Z. Yuan, Y. Yin, and H. Pan. Study on the linear dynamic model of shield TBM cutterhead driving system, IECON 201137th Annual Conference of the IEEE Industrial Electronics Society, pp. 3864-3871, 2011.

[24] Z. Liu, J. Xu, Q. Cheng, Y. Zhao, and C. Yang. Trajectory planning with minimum synthesis error for industrial robots using screw theory, International Journal of Precision Engineering and Manufacturing, vol. 19, no. 2, pp. 183-193, 2018.

[25] S. Wu, M. Wang, Y. Zou. Bidirectional cognitive computing method supported by cloud technology, Cognitive Systems Research, vol. 52, pp. 615-621, 2018, doi:10.1016/j.cogsys.2019.03.015.

[26] D. Di Vito, C. Natale, and G. Antonelli. A comparison of damped least squares algorithms for inverse kinematics of robot manipulators, IFAC-PapersOnLine, vol. 50, no. 1, pp. 6869-6874, 2017, doi:10.1016/j. ifacol.2017.08.1209.

[27] S. Pellegrinelli, A. Orlandini, N. Pedrocchi, A. Umbrico, and T. Tolio. Motion planning and scheduling for human and industrial-robot collaboration, CIRP Annals, vol. 66, no. 1, pp. 1-4, 2017, doi:10.1016/j. cirp.2017.04.095.

[28] S. Wu, Nonlinear information data mining based on time series for fractional differential operators, Chaos, vol. 29, pp. 013114, 2019, doi:10.1063/1.5085430.

[29] L. Ji, H. Wang, T. Zheng, and X. Qi. Motion trajectory of human arms based on the dual quaternion with motion tracker, Multimedia Tools and
Applications, vol. 76, no. 2, pp. 1681-1701, 2017, doi:10.1007/s11042-0153099-y.

[30] J. Xu, H. Yang, M. Liu, J. Tian, and B. Liu. Research on winding trajectory planning for elbow pipe based on industrial robot, The International Journal of Advanced Manufacturing Technology, vol. 93, no. 1-4, pp. 537545, 2017, doi:10.1007/s00170-017-0568-7.

[31] R. Walambe, N. Agarwal, S. Kale, and V. Joshi. Optimal trajectory generation for car-type mobile robot using spline interpolation, IFAC-PapersOnLine, vol. 49, no. 1, pp. 601-606, 2016, doi:10.1016/j. ifacol.2016.03.121.

[32] H. M. Alwan, and Z. H. Rashid. Motion Control of Three Links Robot Manipulator (Open Chain) with Spherical Wrist, Al-Khwarizmi Engineering Journal, vol. 15, no. 2, pp. 13-23, 2019, doi:10.22153/ kej.2019.12.001.

[33] K. Leng, Y. Bi, L. Jing, H. Fu, I. Van Nieuwenhuyse. Research on agricultural supply chain system with double chain architecture based on blockchain technology, Future Generation Computer System, vol. 86, pp. 641-649, 2018, doi:10.1016/j.future.2018.04.061.

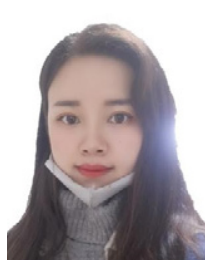

\section{Li You}

Li You Master of Fine Art currently a lecturer of Art and Design School in Wuchang Institute of Technology. Her research interests include digital media art, comprehensive material painting and animation modeling research. Dozens of her academic papers and paints have been published in Chinese Journals as well as international Journals. Email:120120962@wut.edu.cn

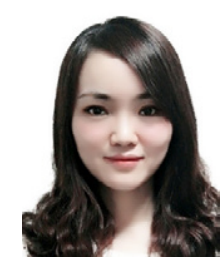

Juan Wang

Juan Wang was born in Zhongxiang, Hubei, P.R. China, in 1987. She received the Master degree from Hubei University of Technology, P.R. China. Now, she works in School of Arts Design, Wuchang University of Technology. Her research interests include animation design and digital illustration design. Her papers and works have won awards in provincial competitions for many times, and she has published many core journals and works. E-mail:120100054@wut.edu.cn

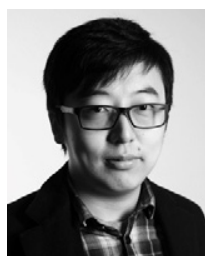

\section{Yiming Ji}

Yiming Ji now works as a lecturer in School of journalism at Zhongnan university of economics and law, Master of fine art. His research interests include digital media art, computer graphics, animation design and cartoon creation. His works have participated in international exhibitions, national exhibitions and provincial exhibitions for many times. Email: jym20200123@163.com 